Lee Jiun

\title{
AUTOLOGOUS UMBILICAL CORD BLOOD CELLS FOR NEWBORN INFANTS WITH HYPOXIC-ISCHEMIC ENCEPHALOPATHY
}

\section{ABSTRACT}

Many of the recent clinical trials have shown high therapeutic potential of cord blood stem cells to treat various diseases in children. In this report we present the results of pilot study of autologous umbilical cord blood cells transplantation for newborn infants with hypoxic-ischemic brain injury. At one year old, case 1 was normal developmentally. Case 2 has gross motor delay with relative sparing of cognition. A multicentre randomised-controlled trial is needed to evaluate clinical effectiveness of this treatment.

KEY WORDS: cord blood, hypoxic-ischemic brain injury, stem cell transplantation.

Hypoxia-ischemia is a dreaded cause of perinatal neurologic injury. Despite advances in maternal-fetal medicine and great strides in prevention, moderate to severe hypoxic-ischemic encephalopathy (HIE) of the newborn baby still occur every 1-2 per 1000 live births. Until very recently the management of such infants largely involves supportive care for major organ dysfunctions. Intensive research in both animals and subsequently humans over the past 3 decades has convincingly shown that induced systemic hypothermia, in which an infant's body temperature is kept at 33,50C starting within 6 hours of birth and maintained for 72 hours, is effective in improving neurological outcome for infants with HIE. This therapy has thus become standard of care since $2010[1,2,3]$. Unfortunately even after treatment with induced hypothermia, a significant proportion of HIE infants still has neurological disabilities of varying degrees when assessed at 2 years of age [4]. Research thus continues in the quest for other modalities that will further improve outcomes.

\section{STEM CELLS IN NEUROLOGICAL DISEASES}

There has been a long history of interest in cell replacement therapy for neurological diseases. Even as far as forty years ago many studies on neuronal implants in animals were carried out [5]. For the purpose of neuroregeneration, the other less studied strategy involves stimulation of endogenous neural stem cells. In Parkinson's disease, two doubleblind randomised controlled clinical trials were performed using transplanted fetal tissue, and the results were promising even though primary endpoints were not achieved [6,7]. What was encouraging was that these trials demonstrated that implanted cells of human embryonic origin were successfully grafted and produced fibre outgrowth. Despite this, big challenges remain in translating these incremental advances into routine therapeutics. Apart from clinical efficacy, the other important consideration when using fetal tissue is medical ethics. Alternative sources of stem cells would be obvious candidates to circumvent controversial political and regulatory hurdles. Such examples include xenogeneic fetal cells, immortalized cell lines, mesenchymal stem cells, bone marrow stromal cells, adipose-derived stem cells, placenta-derived stem cells, umbilical cord blood cells and induced pluripotent stem cells [8].

\section{UMBILICAL CORD BLOOD CELLS}

Umbilical cord blood is a rich source for stem cells, including mesenchymal and monocyte-derived stem cells. Human umbilical cord blood (HUCB) cell transplant was first used in 1988 to successfully treat a child with Fanconi anemia, despite much initial skepticism. It is now a well-accepted treatment option for many blood cancers and genetic hematologic diseases. By 2015 it is estimated that 10000 cord blood transplant will be performed annually around the world using publicly stored cord blood. There are many clear advantages of using HUCB as a source of pluripotent stem cells. It is normally discarded as biological waste after an infant's delivery, easily accessible, and its collection and storage is risk-free for the donor. Due to its low immunogenicity HUCB cell transplants are also more tolerant of HLA mismatches and thus have a lower incidence of graft-versus-host disease when compared to bone marrow stem cells. Problems of using HUCB in transplant include quantity of cells available from a single donor when used in a large patient.

In animal models with induced stroke, HUCB cell infusion demonstrated some important and consistent features: infused cells migrated to areas of injury, reduced the size of the infarcted area, and caused functional improvements in the animals' neurological scores [9, $10,11]$. In humans there was also proof ina patient with Krabbe disease that intravenously infused umbilical cord blood cells crossed the bloodbrain barrier and engrafted in the recipient's brain tissue. [J. Kurtzberg, personal communication]. 


\section{POSSIBLE MECHANISMS FOR THE BENEFICIAL NEUROLOGICAL EFFECT OF INFUSED STEM CELLS}

Contrary to common logic, transplanted cells probably do not exert their benevolent effect in a major way via reproduction, differentiation and replacing neural cells lost to disease [12]. Paracrine effect through secreted neurotrophic factors that help stimulate endogenous neural precursor reproduction and function is one possible mechanism $[13,14]$. HUCB protected cortical neurons against glutamate-induced apoptosis by activation of the protein kinase B signaling pathway [15]. This is especially pertinent to HIE therapeutics because preventing delayed cell death after an acute hypoxic-ischemic insult is the underlying basis for successful treatment in infants with HIE. HUCB also improved oligodendrocyte survival through alterations in gene expression [16] In a rat model of spinal cord injury transplanted HUCB multipotential stem cells improve neurologic function possibly by the release of neurotrophic, angiogenic, and anti-inflammatory cytokines that reduce secondary injury [17]. Mononuclear cells derived from HUCB were shown to secrete interleukins, growth factors, and chemotactic proteins in in-vitro experiments [18].

\section{AUTOLOGOUS UMBBILICAL CORD BLOOD CELLS FOR NEWBORN INFANTS WITH HYPOXIC- ISCHEMIC BRAIN INJURY - A PILOT STUDY}

This is an attractive idea because the immediate availability of autologous cord blood after delivery of an infant meant that the benefit of any infused stem cell would likely be greatest soon after the injury has occurred, especially during the reperfusion phase. In addition there would be no problem of HLA incompatibility, and the nucleated cell viability of fresh cord blood would be better than cryopreserved cord blood units. Thus in collaboration with Cotten et al. from Duke University who had initiated a pilot study on autologous cord blood cells for infants with HIE, a similar pilot study on safety and feasibility was carried out in Singapore $[19,20]$. Over a period of 2 years, infants with HIE and who required therapeutic hypothermia were eligible for autologous cord blood transfusion. The collected cord blood unit would be volume and red blood cell-reduced to obtain the nucleated cell fraction. It would not be cryopreserved, and the nucleated cell fraction would be divided into 4 aliquots of $5 \mathrm{ml}$ each. Each aliquot would be infused at 12, 24, 48 and 72 hours of age. We demonstrated that for non-cryopreserved cord blood nucleated cell fractions, the post-processing cell viability at 96 hours was 91,3\% (CD34+ fraction 93,3\%). Two infants were enrolled. Both had Sarnat stage 2 HIE and seizures. Autologous cord blood was infused as planned (6 and 156 million cells/kg birth weight for each infant). There were no adverse reactions related to the transfusions. Both infants had MRI brain abnormalities initially but second MRIs at 4-6 month of age were normal. At one year old, case 1 was normal developmentally. Case 2 has gross motor delay with relative sparing of cognition. A multicentre randomised-controlled trial is needed to evaluate clinical effectiveness of this treatment. Anticipated challenges of such a trial will be the ethics of having a placebo control group, as well as the possible difficulties in collecting sufficient volumes of cord blood especially if prior informed consent is required from parents. An accredited public cord blood bank in close proximity to birthing hospitals is also necessary to produce good quality cell fractions and ensure timely infusions.

\section{REFERENCES}

1. Gunn A. et al. Dramatic neuronal rescue with prolonged selective head cooling after ischemia in fetal lambs // J. Clin. Invest. - 1997. - 99, № 2. - P. 248 .

2. Edwards A. et al. Neurological outcomes at 18 months of age after moderate hypothermia for perinatal hypoxic ischaemic encephalopathy: synthesis and metaanalysis of trial data // BMJ: British Medical Journal. - 2010. - 340. - P. 363

3. Perlman J. et al. Neonatal resuscitation: 2010 international consensus on cardiopulmonary resuscitation and emergency cardiovascular care science with treatment recommendations // Pediatrics. - 2010. - 126, № 5. - P. 1319-1344.

4. Jacobs $S$. et al. Whole-body hypothermia for term and near-term newborns with hypoxic-ischemic encephalopathy: a randomized controlled trial // Arch. Pediat. Adol. Med. - 2011. - 165, № 8. - P. 692.

5. Das G., Altman J. Transplanted precursors of nerve cells: their fate in the cerebellums of young rats // Science. - 1971. - 173, № 3997 . - P. 637-638.

6. Freed C. et al. Transplantation of embryonic dopamine neurons for severe Parkinson's disease // New Engl. J. Med. - 2001. - 344, № 10. - P. 710-719.

7. Olanow C. et al. A double-blind controlled trial of bilateral fetal nigral transplantation in Parkinson’s disease // Ann. Neurol. - 2003. - 54, № 3. - P. 403-414.

8. Trounson A. et al. Clinical trials for stem cell therapies // BMC Med. - 2011. - 9, № 1. - P. 52.

9. Chen J. et al. Intravenous administration of human umbilical cord blood reduces behavioral deficits after stroke in rats // Stroke. - 2001. - 32, № 11. P. 2682-2688.

10. Meier C. et al. Spastic paresis after perinatal brain damage in rats is reduced by human cord blood mononuclear cells // Pediat. Res. - 2006. - 59, № 2. P. 244-249

11. Pimentel-Coelho P. et al. Human cord blood transplantation in a neonatal rat model of hypoxic-ischemic brain damage: functional outcome related to neuroprotection in the striatum // Stem Cells Dev. - 2010. - 19, № 3. - P. 351-358.

12. Snyder E. et al. Multipotent neural precursors can differentiate toward replacement of neurons undergoing targeted apoptotic degeneration in adult mouse neocortex // Proc. Nat. Acad. Sci. - 1997. - 94, № 21. - P. 11663-11668.

13. Ourednik J. et al. Neural stem cells display an inherent mechanism for rescuing dysfunctional neurons // Nat. biotechnol. - 2002. - 20, № 11. - P. 1103-1110.

14. Ben-Shaanan T., Ben-Hur T., Yanai J. Transplantation of neural progenitors enhances production of endogenous cells in the impaired brain // Mol. Psychiatr. 2007. - 13, № 2. - P. 222-231.

15. Dasari V. et al. Neuroprotection by cord blood stem cells against glutamate-induced apoptosis is mediated by Akt pathway // Neurobiology of disease. 2008. - 32, № 3. - P. 486-498.

16. Rowe D. et al. Cord blood administration induces oligodendrocyte survival through alterations in gene expression // Brain res. -2010 . - 1366. $-\mathrm{P}$. 172-188.

17. Chua S. et al. The effect of umbilical cord blood cells on outcomes after experimental traumatic spinal cord injury // Spine. - 2010. - 35, № 16. - P. 1520-1526.

18. Neuhoff S. et al. Proliferation, differentiation, and cytokine secretion of human umbilical cord blood-derived mononuclear cells in vitro // Exp. Hematol. 2007. - 35, № 7. - P. 1119-1131.

19. Liao Y. et al. Rescuing the neonatal brain from hypoxic injury with autologous cord blood // Bone Marrow Transplantation. - 2013. - 48, №7. - P. 890-900.

20. National University Hospital, Singapore. Autologous cord blood cells for brain injury in term newborns. In: ClinicalTrials.gov. Bethesda (MD): National Library of Medicine (US). 2000 - NLM Identifier: NCT01649648 [cited 2013 Sep 03]. - Available from: http://clinicaltrials.gov/show/NCT01649648. 